THE death is announced of Dr. Benedict Wallet Vilakazi, the eminent Zulu poet, who won a prize in the competition for literary works in African vernacular languages organized by the International African Institute in 1932 . He became assistant lecturer in Bantu languages in the University of Witwatersrand and was the first African in the Union to receive the degree of Doctor of Letters. At his funeral service Dr. C. Doke, his colleague and chief at the University, paid a notable tribute to his qualities. 'Vilakazi', he said, 'was obsessed with a great desire for the intellectual uplift of his people. He believed in a great and honourable future for the literature of the Bantu.... He has set before his people a great example.' We had the pleasure of printing in Africa his beautiful poem on the Victoria Falls.

\title{
A New Appointment
}

DR. S. F. NADEL, whose name and work are familiar to readers of this Journal, has been appointed Reader in Social Anthropology at Durham University. We hope to publish in our next number a review of his new book, The Nuba.

\section{Les Sciences humaines à Madagascar}

Nous sommes reconnaissants à M. J. Faublée pour la note suivante:

A Madagascar, l'organisation des recherches de sciences humaines commence seulement. Depuis la réunion de Madagascar à la France métropolitaine l'Académie malgache dut assurer un rôle de coordination. L'Académie est une réunion de malgachisants ayant publié au moins une étude sur un sujet malgache, choisis par cooptation. Elle publie un bulletin et des mémoires. Il est à noter que la plupart des membres sont européens. Des lettrés malgaches sont membres, mais publient rarement leurs travaux, interviennent peu dans les séances. Les malgachisants âgés qui dirigent la société sont remarquables par leur connaissance des anciennes coutumes. Ils s'intéressent à une ethnographie archaïque, évoquent les faits passés et l'ancienne société, négligènt de décrire l'évolution actuelle. La plupart demeurent à Tananarive; des régions échappent à toute étude.

La guerre empêcha de fonctionner normalement le service de la recherche scientifique. Monsieur Decary, qui le dirigeait, dut se limiter à quelques enquêtes, à coordonner les efforts des missions spécialisées envoyées de la métropole et ceux des chercheurs demeurant sur place. Son ambition était d'obtenir des monographies tribales homogènes et comparables. Sur les dix-huit tribus de Madagascar quatre groupes du Sud seulement ont fait l'objet de monographies assez détaillées. Au cours d'un séjour à Madagascar, de 1938 à I94I, je m'aperçus des difficultés de l'établissement de monographies tribales. Il y a trop peu de missions d'ethnographes professionnels pour les faire. Ceux qui résident sur place sont trop souvent limités à un domaine géographique trop restreint à l'intérieur d'une tribu. Pour eux une tribu est un domaine trop vaste, tandis qu'ils sont les mieux placés pour établir des monographies de villages. D'autre part la monographie a des défauts d'exposition: elle place sur des plans équivalents des faits d'importance inégale. Des techniques secondaires comme le lavage du linge, par exemple, prennent une importance trop grande.

Monsieur Decary organisa le dépouillement des derniers recensements. Il en résulta un travail important qu'il publia en collaboration avec Monsieur Castel: Modalités et conséquences des migrations intérieures récentes des populations malgaches. Cet ouvrage complète une carte ethnographique et démographique. Ces travaux mettent en valeur le fait capital de l'ethnographie de Madagascar: la population est tellement mêlée par les migrations que les anciens cadres tribaux ne peuvent servir de limites à un travail. Il est maintenant impossible 
d'étudier, par exemple, les pasteurs Bara du Sud sans tenir compte des cultivateurs immigrants du Nord, de l'Est, du Sud. Par ailleurs, les tribus anciennes ayant une base dynastique et non territoriale, il est difficile de les étudier par zones géographiques.

Tandis que l'Académie malgache et le Service de la recherche scientifique poursuivaient ces travaux, des chercheurs indépendants travaillaient dans une autre ligne. Je citerai avant tout le travail d'une équipe de Malgaches dirigés par l'un d'eux: Ranjavula. Ils entreprirent en 1937 la rédaction et la publication d'une encyclopédie malgache en malgache et les poursuivent avec une ténacité digne d'éloges.

Le service de la recherche scientifique fut réorganisé en 1947. Il est dirigé par le Docteur Millot, professeur au Museum national d'Histoire Naturelle.

Ce n'est que sous l'angle sociologique que l'on fera admettre l'ethnographie aux Malgaches. Pour eux c'est l'étude du passé, c'est les considérer en sauvages ou en primitifs. S'ils aident aussi peu à l'étude de leur monde, c'est en raison de cette réserve. Présentée comme une sociologie, l'étude de leur société sera sur le même plan que l'étude d'une société européenne. Le service de la recherche leur demandera des autobiographies par exemple. Des rédactions sur des sujets malgaches seront demandées aux instituteurs et à leurs élèves. En principe les réponses seraient en langue malgache. Les premiers coenquêteurs et informateurs seront probablement des Malgaches évolués, européanisés. Mais il est à prévoir des enquêtes analogues dans les sociétés restées relativement archaïques.

Actuellement il se produit une coupure dangereuse entre Malgaches traditionalistes et Malgaches évolués. Ces derniers sont séparés des premiers, parfois dans la même famille, par l'oubli des coutumes anciennes, et surtout de la langue malgache. Une collection de textes traditionnels est prévue parmi les publications du service. Le but de cette édition est multiple: les textes seront traduits en français et les volumes publiés sous deux formes: texte bilingue, textes séparés. Des Européens utiliseront les traductions comme documents sociologiques, sans pouvoir se reporter au texte original, ou les considéreront comme les œuvres d'art qu'elles reproduisent. D'autres les emploieront à apprendre le malgache. Des Malgaches y étudieront le français, tandis que d'autres liront en leur langue ces contes qui ont été la sagesse des ancêtres. Bon nombre de Malgaches savent lire. Mais à part des quotidiens à la littérature souvent médiocre, à part des livres chrétiens, ils n'ont rien à lire. En éditant à bon compte des textes malgaches le service de la recherche n'accomplira pas seulement une œuvre scientifique, mais encore plus une œuvre sociale. Publiant récemment une anthologie de leur littérature, deux Malgaches lettrés ont montré que, trop souvent, ils n'avaient pu lire que les traductions françaises de leur propre langue.

Actuellement si un Malgache ignore un mot de sa langue - et celle-ci est riche-il le cherche dans un dictionnaire malgache-français ou malgache-anglais, et s'il ne comprend pas la traduction européenne, il n'a pas d'autre ressource. Des Malgaches ont rédigé des dictionnaires tout en malgache. Le service de la recherche aidera à leur publication. Les dictionnaires malgaches actuels sont insuffisants. La mise au point d'un dictionnaire détaillé, étoffé d'exemples, est un des premiers travaux qu'entreprendra le service.

\section{Ordonnance legislative no. 157}

As this number is going to press we learn that the Ordinance of which Mr. Davidson writes on page 127 has been withdrawn from the Belgian Colonial Council, and a new one is being prepared. 